\title{
Características del líquido cefalorraquídeo en niños con infecciones del sistema de derivación ventrículo-peritoneal
}

\author{
Characteristics of cerebrospinal fluid (CSF) among children with \\ ventriculoperitoneal shunt infections
}

\author{
Dr. Hernán Rowensztein ${ }^{a}$ Dr. Lisandro Manfrin ${ }^{a}$, Dra. Marcela Paglia ${ }^{a}$ Dr. Tai Luong Cong ${ }^{a}$, \\ Dra. Silvina Ruvinsky y Dra. Adriana Scrigni ${ }^{b}$
}

\begin{abstract}
RESUMEN
Una complicación frecuente (5\%) de las derivaciones de líquido cefalorraquídeo (LCR) es la infección. Se han utilizado las características citoquímicas del LCR para decidir el tratamiento antimicrobiano empírico inicial. El objetivo fue investigar las características citoquímicas y microbiológicas que presentan los LCR de niños con pioventriculitis asociadas a sistemas de derivación ventrículo-peritoneales. Se realizó un estudio retrospectivo, descriptivo y analítico. Se incluyeron 32 casos de pioventriculitis; predominaron las bacterias Gram positivas con 26 casos ( $81,25 \%)$; el resto fueron gérmenes Gram negativos. No hubo diferencias estadísticamente significativas para las variables estudiadas (glucorraquia, proteinorraquia $\mathrm{y}$ celularidad).

Conclusión: las bacterias Gram positivas predominaron ampliamente como causa de pioventriculitis asociada al acto quirúrgico. Las características del LCR muestran alta heterogeneidad y no se asociaron con el tipo de germen en esta muestra estudiada.

Palabras clave: ventriculitis cerebral, derivación ventrículoperitoneal, líquido cefalorraquídeo, hidrocefalia.
\end{abstract}

http:/ /dx.doi.org/10.5546/aap.2015.244

\section{INTRODUCCIÓN}

El exceso de producción de líquido cefalorraquídeo (LCR) y/o la obstrucción en su circulación producen hidrocefalia ${ }^{1}$ y son las causas más comunes de requerimientos de colocación de derivaciones de LCR. Estas son cada vez más utilizadas en pediatría, debido

a. Servicio de Clínica Pediátrica en Cuidados Intermedios y Moderados.

b. Servicio de Epidemiología e Infectología.

Hospital de Pediatría "Prof. Dr. Juan P. Garrahan"

de Buenos Aires, Argentina.

Correspondencia:

Dr. Hernán Rowensztein: hrowens@intramed.net

Financiamiento: Ninguno.

Conflicto de intereses: Ninguno que declarar.

Recibido: 7-7-2014

Aceptado: 4-12-2014 a la supervivencia de niños prematuros que sufren hemorragias intraventriculares. También se continúan utilizando para otros tipos de hidrocefalias, como las secundarias a tumores del sistema nervioso central (SNC), hidrocefalias congénitas, etc.

Una complicación temida y frecuente es la infección del sistema de derivación vinculada al acto quirúrgico de colocación, que oscila entre el 0 y el $20 \%$ según la serie. ${ }^{2}$ Debido a que el cultivo definitivo del LCR puede demorar entre 5 y 7 días, habitualmente, en la práctica clínica, se han utilizado las características citoquímicas del LCR para decidir el tratamiento antimicrobiano empírico inicial teniendo en cuenta estudios previos publicados. ${ }^{3-5}$

El objetivo del presente trabajo fue investigar las características citoquímicas y microbiológicas que presentan los LCR de niños con pioventriculitis asociadas a sistemas de derivación ventrículo-peritoneales (DVP) y determinar la asociación entre los hallazgos citoquímicos y el tipo de germen.

\section{MATERIALES Y MÉTODOS}

Estudio retrospectivo, descriptivo y analítico, realizado en el Hospital de Pediatría "Prof. Dr. Juan P. Garrahan", centro del tercer nivel de atención ubicado en Buenos Aires, Argentina. Se revisaron los registros médicos (historias clínicas y bases de datos computadas) en el período comprendido entre enero de 2010 y diciembre de 2012. Se seleccionaron todos los pacientes con diagnóstico de pioventriculitis vinculada al acto quirúrgico. Tomamos para esto la definición de ventriculitis del Centers for Disease Control and Prevention's National Healthcare Safety Network (CDC/NHSN) 2014 y que la infección fuera diagnosticada dentro de los 6 meses de la cirugía de colocación del sistema de derivación de LCR. ${ }^{6}$ Todos los LCR fueron obtenidos por punción del reservorio valvular; se recopilaron las 
características físico-químicas y microbiológicas de los LCR al momento del diagnóstico de la pioventriculitis.

Se registraron los datos en una planilla tipo Excel y luego fueron procesados con el paquete estadístico S.P.S.S. v17.0. Se realizaron histogramas para determinar la distribución de las variables numéricas. Según ellos, fueron comparados los grupos utilizando la prueba de Mann-Whitney, dada su distribución no paramétrica. Se determinó la frecuencia de aparición de cada germen; se los agrupó, a su vez, en Gram positivos y negativos; se comparó la celularidad, glucorraquia y proteinorraquia entre los grupos. Se tomó como valor de corte para diferencias estadísticamente significativas un valor de $\mathrm{p}$ menor de 0,05 .

\section{RESULTADOS}

Se incluyeron 32 casos (17 varones) de pioventriculitis documentadas por aislamiento microbiológico en el período estudiado. Esto representa aproximadamente el 5\% del total de DVP colocadas en igual período (640 cirugías). Las edades de los pacientes se encontraban en rango de 3 meses a 13 años.

Los diagnósticos de base de los pacientes, y que originaron la necesidad de colocación de la DVP, fueron tumores del SNC $(n=10)$, hidrocefalia congénita $(n=9)$, hemorragia intraventricular $(n=5)$, malformación de Dandy Walker $(n=2)$ y otros, como meningitis, trauma de cráneo, etc.

En cuanto a la microbiología, todos los cultivos fueron monomicrobianos. Predominaron las bacterias Gram positivas con 26 casos $(81,25 \%)$; el resto fueron gérmenes Gram negativos; no se hallaron hongos. Las bacterias más frecuentemente halladas fueron los estafilococos coagulasa negativos meticilino resistentes $(n=17)$, seguidos por Staphylococcus aureus meticilino sensible $(n=5)$ y el meticilino resistente $(n=3)$ y luego por Pseudomonas aeruginosa $(\mathrm{n}=3)$ y Acinetobacter $(\mathrm{n}=2)$; el resto corresponde a otros gérmenes.

Se compararon las medianas y los rangos de dispersión para la glucorraquia, proteinorraquia y número de glóbulos blancos en el LCR -discriminados según la tinción de Gram-, los cuales se pueden analizar en la Tabla 1. No se hallaron diferencias estadísticamente significativas para las variables estudiadas.

Se analizó también el tiempo transcurrido desde el acto quirúrgico de colocación del sistema de derivación de LCR y el diagnóstico de la pioventriculitis. La mediana en el grupo con gérmenes Gram negativo fue de 27 días (rango 4-139), mientras que, en el grupo Gram positivo, fue de 28 días (rango 2-151), sin diferencias estadísticamente significativas $(p=0,9)$. También analizamos la frecuencia de aparición de gérmenes en los casos en los que la infección se detectó dentro de los 15 días posteriores a la colocación del sistema de DVP y hallamos que esto ocurrió en 11 de las infecciones por gérmenes Gram positivos (42\%) y en la mitad de las infecciones por Gram negativos $(n=3)$, sin diferencias estadísticamente significativas (prueba de Fisher a dos colas=1).

\section{DISCUSIÓN}

La infección es una de las complicaciones más frecuentes de las derivaciones ventriculares de LCR. En relación con los gérmenes que producen pioventriculitis, se observa, en nuestro hospital, un predominio de gérmenes Gram positivos y una disminución de la incidencia de gérmenes Gram negativos. Existen instituciones hospitalarias que coinciden con nuestra tendencia, como señala el artículo de Kuo Wei, ${ }^{7}$ y, en cambio, otras difieren e informan como agente etiológico más común al Acynetobacter (Brasil y Australia). ${ }^{2}$

En el estudio de Grandi L. y colaboradores realizado en nuestro centro y publicado en $1997,{ }^{3}$ se había documentado una significativa diferencia en el número de células que provocaba la infección por gérmenes Gram negativos, que era mayor que en las infecciones por gérmenes Gram positivos. También fue descrito el mismo fenómeno en drenajes de LCR al exterior. ${ }^{5}$ Lo mismo se observó en otros estudios publicados, ${ }^{8-10}$ pero esto no ha podido ser corroborado por nuestra investigación a pesar de existir una tendencia en este sentido en los valores hallados. Estas diferencias quizás se deban a que el número de bacterias Gram negativas ha disminuido notablemente como gérmenes productores de pioventriculitis desde un 30\% (en el período 19921995) al 19\% actual. Además, en el mencionado estudio, han asumido una distribución normal de las variables y, por lo tanto, usado pruebas paramétricas para su comparación; mientras que nosotros hemos usado pruebas no paramétricas (Mann-Whitney) debido a la distribución muy sesgada de las variables estudiadas.

Rosanova y Paganini ${ }^{11}$ señalan que un alto recuento de células en LCR se correlaciona con infección, pero un recuento normal no lo 
invalida. Lo mismo ocurre con la alteración en la glucorraquia y proteinorraquia. Además, debe tenerse en cuenta que está descrito que, en los 15 días posteriores a la colocación de un sistema de DVP, se observa un aumento de la celularidad del LCR por el mismo trauma quirúrgico.

Como se puede observar en la Tabla 1, tanto para gérmenes Gram negativos como Gram positivos, el rango de celularidad en el LCR abarca algunos casos en que es menor de 5, valor tomado como punto de corte para la normalidad. Esto es importante dado que, entonces, habría casos de pioventriculitis con celularidad normal en el LCR que requerirían de una gran sospecha diagnóstica para comenzar el tratamiento empírico, como ocurre en el 11\% de los casos del estudio publicado por Von der Brelie. ${ }^{12}$

El estudio de Fulkerson y colaboradores ${ }^{10}$ halló diferencias importantes en el recuento de glóbulos blancos del LCR dependiendo del tipo de bacteria, pero llama la atención la gran celularidad hallada tanto para gérmenes Gram positivos como Gram negativos. Al igual que en nuestro estudio, no está descrito el tiempo de evolución desde el comienzo de los síntomas al momento del diagnóstico de la infección (por la dificultad de obtener este dato en estudios retrospectivos), que, de ser este tiempo muy prolongado, podría ser una de las causas de este importante incremento en la celularidad.

También se ha reportado, en algunas series, un aumento de los eosinófilos en el LCR de los niños con pioventriculitis, aunque este hallazgo sea poco frecuente. Los eosinófilos en el LCR se describieron asociados -además de a infeccionesa extravasación y presencia de sangre en el LCR, alergias a siliconas y otros materiales. ${ }^{13,14} \mathrm{Se}$ considera eosinofilia la presencia de, al menos, un eosinófilo por campo, ya que no deberían estar presentes. En nuestra revisión, no se hallaron

TABla 1. Características del líquido cefalorraquídeo en pioventriculitis de acuerdo con la tinción de Gram $(n=32)$

\begin{tabular}{lccc}
\hline & $\begin{array}{c}\text { Gram } \\
\text { positivos }^{1}\end{array}$ & $\begin{array}{c}\text { Gram } \\
\text { negativos }^{1}\end{array}$ & P $^{*}$ \\
\hline Glucorraquia mg/dl & $\begin{array}{c}49,5 \\
(0-96)\end{array}$ & $\begin{array}{c}16 \\
(0-84)\end{array}$ & 0,14 \\
& 54,5 & 117,5 & 0,17 \\
Proteinorraquia mg/dl & $(10-684)$ & $(10-320)$ & \\
& 21 & 70 & 0,79 \\
Glóbulos blancos/mm $\mathrm{mm}^{3}$ & $(0-1900)$ & $(2-240)$ & \\
\hline * Mann-Whitney. & & & \\
${ }^{1}$ Mediana (rango). & & &
\end{tabular}

pacientes con esta característica.

Otra variable que se ha estudiado previamente para poder predecir el germen que pudiera estar provocando la pioventriculitis es el tiempo transcurrido entre la colocación del sistema y la aparición de la infección, pero tampoco se ha demostrado su utilidad en nuestra investigación. El artículo de McGirt ${ }^{15}$ coincide con nuestros resultados: las bacterias diagnosticadas antes de los 14 días de colocada la DVP eran las mismas que pasado ese período.

Una de las debilidades en nuestra investigación que se repite en los trabajos encontrados es el escaso número de pacientes que presentan esta infección y, por lo tanto, el pequeño tamaño muestral que puede influir en el poder estadístico para hallar diferencias entre los grupos. Otra limitación del presente trabajo es ser retrospectivo, lo que restringe el análisis de ciertas variables como sintomatología de los pacientes.

\section{CONCLUSIÓN}

Las bacterias Gram positivas predominaron ampliamente como causa de pioventriculitis asociada al acto quirúrgico. A diferencia de estudios anteriores, las características del LCR muestran alta dispersión de los valores y no se asociaron con el tipo de germen en esta muestra estudiada. Por el momento, el citoquímico del LCR no sería orientador del diagnóstico microbiológico ni sería útil para poder decidir el tratamiento antibiótico empírico inicial.

\section{REFERENCIAS}

1. Sakka L, Coll G, Chazal J. Anatomy and physiology of cerebrospinal fluid. Eur Ann Otorhinolaryngol Head Neck Dis 2011;128(6):309-16.

2. Stenehjem E, Armstrong WS. Centralnervous system device infections. Infect Dis Clin North Am 2012;26(1):89-110.

3. Grandi L, Goldsman A, Jaimovich J, Cohen Arazi J. Pioventriculitis asociada a derivaciones ventriculares. Med Infant 1997;4(1):7-10.

4. Lee P, DiPatri AJ. Evaluation of suspected cerebrospinal fluid shunt complications in children. Clin Pediatr Emerg Med 2008;9(2):76-82.

5. Pfisterer W, Mühlbauer M, Czech T, Reinprecht A. Early diagnosis of external ventricular drainage infection: results of a prospective study. I Neurol Neurosurg Psychiatry 2003;74(7):929-32.

6. Centers for Disease Control and Prevention. CDC/NHSN Surveillance definitions for specific types of infections. Atlanta: CDC; 2014. [Acceso: 4 de diciembre de 2014]. Disponible en: http://www.cdc.gov/nhsn/PDFs / pscManual/17pscNosInfDef_current.pdf.

7. Wang KW, Chang WN, Shih TY, Huang CR, et al. Infection of cerebrospinal fluid shunts: causative pathogens, clinical features, and outcomes. Jpn J Infect Dis 2004;57(2):44-8.

8. McClinton D, Carraccio C, Englander R. Predictors of ventriculoperitoneal shunt pathology. Pediatr Infect Dis J 2001;20(6):593-7. 
9. Muttaiyah S, Ritchie S, Upton A, Roberts S. Clinical parameters donot predict infection in patients with external ventricular drains: a retrospective observational study of daily cerebrospinal fluid analysis. J Med Microbiol 2008;57(Pt 2):207-9.

10. Fulkerson DH, Sivaganesan A, Hill JD, Edwards JR, et al. Progression of cerebrospinal fluid cell count and differential over a treatment course of shunt infection. J Neurosurg Pediatr 2011;8(6):613-9.

11. Rosanova MT, Paganini H. Infecciones asociadas a sistemas de derivación de líquido cefalorraquídeo. Med Infant 1997;4(1):26-30

12. Von der Brelie C, Simon A, Gröner A, Molitor E, et al.
Evaluation of an institutional guideline for the treatment of cerebrospinal fluid shunt-associated infections. Acta Neurochir (Wien) 2012;154(9):1691-7.

13. Bezerra S, Frigeri TM, Severo CM, Santana JC, et al. Cerebrospinal fluid eosinophilia associated with intraventricular shunts. Clin Neurol Neurosurg 2011;113(5):345-9.

14. Fulkerson DH, Boaz JC. Cerebrospinal fluid eosinophilia in children with ventricular shunts. J Neurosurg Pediatr 2008;1(4):288-95.

15. McGirt MJ, Zaas A, Fuchs HE, George TM, et al. Risk factors for pediatric ventriculoperitoneal shunt infection and predictors of infectious pathogens. Clin Infect Dis 2003;36(7):858-62.

\title{
Archivos hace 75 años
}

Año XI Mayo de $1940 \quad$ Tomo XIII, N • 5

\section{ARCHIVOS ARgENTINOS DE PEDIATRÍA}

PUBLIOAOIÓN MENSUAL

(Organo Oficial de la Sociedad Argentina de Pediąría)

Hospital de Niños - Servicio de Neuropsiquiatría y Endocrinología

El sindrome depresivo melancólico en la infancia (*)

por los doctores

\begin{abstract}
Aquiles Gareiso, Pedro Osvaldo Sagreras y Alejandro J. Petre
\end{abstract}
El tema que vamos abordar, lo consideramos de real-interés, si tenemos presente la tendencia que tiene el pediatra, a derivar estos enfermitos hacia el especialista para su estudio y tratamiento, pues conceptuamos que el examen psíquico del niño debe interesarlo, tanto como el estudio de sus diferentes aspectos físicos, a fin de despistar y tratar estas afececiones, tan frecuentes hoy por desgraeia. Es así, que hacemos propia la afirmación de Rott, M. E., de Berlín, en la segunda conferencia de la Asociación Internacional de Pediatría Preventiva realizada en Ginebra, del 28 al 29 de septiembre de 1932. Decía el citado facultativo: "Los pediatras deberán ocuparse del psiquismo del niño, tanto como de lo físico. El niño difícil de aprender o instruir es un enfermo. Despistar, tratar, y sobre todo prevenir las psicopatías, vá más allá del médico de familia, es una tarea social. La falta de trabajo de los padres por ejemplo, tiene una repercusión sobre el estado nervioso de los mismos y de los hijos".

El texto completo se encuentra disponible en la versión electrónica de este número. 